\title{
FACTORES ASOCIADOS A LA NO UTILIZACIÓN DE LOS SERVICIOS FORMALES DE PRESTACIÓN EN SALUD EN LA POBLACIÓN PERUANA: ANÁLISIS DE LA ENCUESTA NACIONAL DE HOGARES (ENAHO) 2015
}

\author{
Vicente A. Benites-Zapata ${ }^{1, a}$, Michelle Lozada-Urbano, ${ }^{2, b}$, Diego Urrunaga-Pastor ${ }^{3, c}$, Edith Márquez-Bobadilla ${ }^{4,5, d}$, \\ Enrique Moncada-Mapelli, ${ }^{3, c}$, Edward Mezones-Holguín ${ }^{6,7, e}$
}

\begin{abstract}
RESUMEN
El objetivo del estudio fue estimar la prevalencia de la no utilización de los servicios formales de prestación de salud (NUSFPS) y sus factores asociados en Perú. Se realizó un análisis secundario de datos de la Encuesta Nacional de Hogares (ENAHO) del año 2015. Se definió como NUSFPS a aquellos participantes que, pese a haber presentado algún síntoma, malestar, enfermedad, recaída de enfermedad crónica o accidente durante el último mes, no acudieron a los servicios de salud. Se analizaron 35036 participantes; la prevalencia de NUSFPS fue de 53,9\% (IC95\%:52,9-54,8). La NUSFPS fue superior en la costa (razón de prevalencia ajustada $[R P a]=1,24 ; \mathrm{IC} 95 \%: 1,17-1,31)$, sierra $(\mathrm{RPa}=1,38 ; \mathrm{IC} 95 \%: 1,31-1,46)$ y selva $(\mathrm{RP}=$ 1,25;IC95\%:1,18-1,33) en comparación a Lima Metropolitana. Hubo mayor prevalencia de NUSFPS en los participantes sin seguro $(\mathrm{RPa}=1,59 ; \mathrm{IC} 95 \%: 1,52-1,66)$ y afiliados al seguro integral de salud $(\mathrm{RPa}=1,16 ; \mathrm{IC} 95 \%: 1,11-1,22)$ comparados con los afiliados a la Seguridad Social. En conclusión, más de la mitad de los participantes padecieron NUSFPS, lo cual se asoció con condiciones geográficas y de aseguramiento. Se sugieren políticas públicas informadas en la evidencia para mejorar esta situación.
\end{abstract}

Palabras clave: Accesibilidad a los Servicios de Salud; Sistemas de Salud; Servicio Ambulatorio en Hospital; Administración de los Servicios de Salud; Seguro; Perú (Fuente: DeCS BIREME).

\section{ASSOCIATED FACTORS TO NON-USE OF FORMAL HEALTH SERVICES IN THE PERUVIAN POPULATION: ANALYSIS OF THE NATIONAL HOUSEHOLD SURVEY (ENAHO) 2015}

\begin{abstract}
The aim of the study was to estimate the prevalence of non-use of health services (NUHS) and its associated factors using the National Household Survey (ENAHO 2015). The participants were defined as NUHS if they have presented any symptoms, discomfort, illness, relapse of chronic illness or accident during the last month and did not go to the health services. 35036 participants were analyzed; the prevalence of NUHS was $53,9 \%$. NUHS was higher in the coastal region (adjusted Prevalence Ratio [aPR] $=1.24 ; 95 \% \mathrm{Cl}: 1.17-1.31$ ), highlands (aPR=1.38;95\% Cl:1.31-1.46) and jungle (aPR=1.25,95\%Cl:1.18-1.33) compared to Lima. Likewise, there were a higher prevalence of NUHS in participants without health insurance $(\mathrm{aPR}=1.59 ; 95 \% \mathrm{Cl}: 1.52-1.66)$ and those affiliated to Ministry of Health insurance $(\mathrm{aPR}=1.16 ; 95 \% \mathrm{Cl}: 1.11-1.22)$ compared to those affiliated to Social Security. More than half of the participants suffered from NUHS, which was associated with geographical and health system conditions. It is required evidenced-informed public policies to improve this situation.
\end{abstract}

Key words: Health Services Accessibility; Health Systems; Outpatient Clinics, Hospital; Health Services Administration; Insurance; Peru (Source: MeSH NLM).

\footnotetext{
Centro de Investigación en Epidemiologia Clínica y Medicina Basada en Evidencias, Instituto de Investigación, Facultad de Medicina Humana, Universidad de San Martin de Porres. Lima, Perú.

Facultad de Salud Pública y Administración, Universidad Peruana Cayetano Heredia. Lima, Perú.

3 Sociedad Científica de Estudiantes de Medicina, Facultad de Medicina Humana, Universidad de San Martin de Porres. Lima, Perú.

Facultad de Ciencias Matemáticas, Universidad Nacional Mayor de San Marcos. Lima, Perú.

Dirección General de Focalización, Ministerio de Inclusión Social. Lima, Perú.

6 Facultad de Ciencias de la Salud, Universidad Peruana de Ciencias Aplicadas. Lima, Perú.

Instituto Videnza. Lima, Perú.

a Médico cirujano, magister en Ciencias en Investigación Epidemiológica; ${ }^{b}$ licenciada en nutrición, magíster en Salud Pública; ${ }^{c}$ estudiante de Medicina;

${ }^{\mathrm{d}}$ licenciada en Estadística; ${ }^{\mathrm{e}}$ médico cirujano, magister en Ciencias en Epidemiología Clínica.

Recibido: 06/05/2017 Aprobado: 06/09/2017 En línea: 29/09/2017
}

Citar como: Benites-Zapata VA, Lozada-Urbano M, Urrunaga-Pastor D, Márquez-Bobadilla E, Moncada-Mapelli E, Mezones-Holguín E. Factores asociados a la no utilización de los servicios formales de prestación en salud en la población peruana: análisis de la Encuesta Nacional de Hogares (ENAHO) 2015. Rev Peru Med Exp Salud Publica. 2017;34(3):478-84. doi: 10.17843/rpmesp.2017.343.2864 


\section{INTRODUCCIÓN}

La no utilización de los servicios formales de prestación de salud (NUSFPS) por parte de la población, resulta ser prioritario para cualquier sociedad y depende de múltiples factores. Se ha descrito su asociación con aspectos demográficos, con la disponibilidad de recursos financieros, con el contar con un seguro de salud y con la oferta disponible (1). Por su parte, la automedicación, la percepción de no considerar los síntomas de gravedad y el temor a ser diagnosticado de una enfermedad grave (2), son también condiciones relacionadas. Dentro de lo multifactorial de lo expuesto, el contexto del sistema de salud es relevante.

El sistema de salud peruano presenta una estructura compleja con distintas instituciones prestadoras y financiadoras que conllevan a que se le caracterice como segmentado y fragmentado ${ }^{(3)}$. Coexisten fuentes de aseguramiento públicas y privadas, donde el Seguro Integral de Salud (SIS) y el Seguro Social en Salud (EsSalud) resaltan como las principales Instituciones Administradoras de Fondos de Aseguramiento en Salud (IAFAS) en el país ${ }^{(3)}$. A ello se debe agregar que, a pesar de las ofertas de aseguramiento, existen aún brechas de cobertura y se evidencian problemas para acceder a servicios de salud formales ${ }^{(4)}$, lo cual se ve acrecentado por la diversidad geográfica, social y cultural del país. Sin embargo, la evidencia publicada al respecto es escasa.

En este contexto, el objetivo del presente estudio fue estimar la prevalencia e identificar los factores asociados a la NUSFPS en una muestra con representatividad nacional. Los resultados podrían servir de base para la formulación de políticas públicas informadas en la evidencia.

\section{EL ESTUDIO}

\section{DISEÑO}

Se llevó a cabo un análisis secundario de datos de la Encuesta Nacional de Hogares (ENAHO) del Instituto Nacional de Estadística e Informática (INEI) para el año 2015.

\section{POBLACIÓN, MUESTRA Y MUESTREO}

La ENAHO tiene un muestreo probabilístico, estratificado, multietápico e independiente. En el área urbana, la unidad primaria de muestreo fue el centro poblado urbano con dos mil y más habitantes; la unidad secundaria fue el conglomerado que posee en promedio 120 viviendas, en tanto que la unidad terciaria fue la vivienda. Se recolectó la información de todos los participantes que residían en las viviendas seleccionadas. En el área rural, las unidades de muestreo primarias fueron de dos tipos: el centro poblado urbano con 500 a menos de dos mil habitantes y el área de empadronamiento rural el cual tiene en promedio 100

\section{MENSAJES CLAVE}

Motivación para realizar el estudio. La utilización de los servicios formales de salud es un elemento cardinal en el ejercicio de los derechos en salud. Sin embargo, su utilización por parte de los ciudadanos puede afectarse negativamente por múltiples factores.

Principales hallazgos. En Perú, más de la mitad de la población adulta con alguna afectación de su salud, no utiliza los servicios formales de salud; lo cual se asoció con condiciones geográficas y de aseguramiento.

Implicancias. La frecuencia de no utilización de servicios de salud es alta, los factores asociados encontrados pueden servir de base para la propuesta de intervenciones de salud informadas en la evidencia.

viviendas; la unidad secundaria fue el conglomerado que tiene en promedio 120 viviendas, y la unidad terciaria fue la vivienda. Para el año 2015, el tamaño de la muestra fue de 33430 viviendas: 20260 urbanas y 13170 rurales, con la inclusión de un total de 119515 personas.

Para el análisis secundario realizado, se incluyeron a los encuestados mayores de 18 años, quienes presentaron en las últimas cuatro semanas algún síntoma o malestar, enfermedad, recaída de enfermedad o accidente. Se excluyeron a 49789 personas por no presentar enfermedad previa durante el último mes, 20190 entrevistados que no consideraron necesario acudir a un centro de salud a pesar de la presencia de alguna dolencia, 380 participantes por haber acudido por atención médica a una farmacia y a un centro médico a la vez y a 105 participantes que no presentaron datos completos de las variables de interés para el estudio. Finalmente, se analizaron a 35036 personas.

\section{VARIABLES Y MEDICIONES}

La variable de respuesta fue la NUSFPS. Se evaluó si el sujeto había sufrido en las últimas cuatro semanas previas a la entrevista, algún síntoma o malestar, enfermedad, recaída de enfermedad o accidente (p402). A aquellos participantes con una respuesta positiva, se les preguntó por el lugar donde acudió para consultar por esta enfermedad, síntoma o malestar y/o accidente (p403). Se definió como NUSFPS a aquellas personas que refirieron no acudir a recibir atención en una institución prestadora de Servicios de salud (IPRESS) del sistema de salud peruano.

Como variables de exposición se incluyeron el género (p207), la edad (p208a), el estado civil (p209), el nivel educativo (p301), la región (dominio), tener algún problema de salud crónico (p401), tener algún tipo de discapacidad (p401), contar con algún seguro de salud (p419) y las razones por las cuales no acudió al centro o establecimiento de salud (p409). 


\section{ASPECTOS ÉTICOS}

La base de datos de ENAHO no presenta identificadores para cada registro, lo cual permite mantener la confidencialidad correspondiente (http://iinei.inei.gob.pe/ microdatos/). La recolección de datos se realizó previo consentimiento verbal por parte de los participantes y no involucró la toma de muestras biológicas.

\section{PLAN DE ANÁLISIS}

Se utilizó el paquete estadístico STATA ® v14.0 (STATA Corporation, College Station, Texas, USA) con el módulo "complex survey data" (svy). Los resultados descriptivos se presentaron en frecuencias absolutas de la muestra y proporciones ponderadas por el muestreo complejo, con sus intervalos de confianza al 95\% (IC95\%). El análisis bivariado, se hizo mediante la prueba de chi cuadrado para muestras complejas, previa evaluación de los supuestos.

Se llevaron a cabo modelos lineales generalizados de la familia de Poisson (crudo y ajustado) para muestras complejas. Se evaluó las posibles relaciones de colinealidad entre variables a fin de poder obtener una adecuada consistencia estadística en el modelo ajustado. El contar con un seguro de salud y un empleo presentaron colinealealidad, por lo cual excluimos la segunda variable del análisis. La medida de asociación reportada fue la razón de prevalencias (RP) con sus respectivos IC95\%.

\section{HALLAZGOS}

\section{ASPECTOS GENERALES}

En el análisis se incluyeron 35036 entrevistados, lo cual representa 9151146 personas en las 25 regiones políticas del Perú. En la Figura 1 se presenta el flujograma de selección de observaciones.

Seis de cada diez participantes fueron mujeres y la edad promedio fue de 48,1 años. El $28,6 \%$ pertenecía a la región de Lima Metropolitana, el 61,7\% padecía de algún problema crónico, solo $7,5 \%$ de los entrevistados contaba con alguna discapacidad y aproximadamente un cuarto de los participantes no tenía un seguro de salud (Tabla 1). La proporción de participantes que presentaron NUSFPS fue de $53,9 \%$ (Figura 1). Las principales razones referidas por las cuales se presentó la NUSFPS, fueron: se autorecetó o repitió una receta anterior $(27,9 \%)$, por falta de tiempo $(25,5 \%)$ y prefirió curarse con remedios caseros $(20,9 \%)$.

\section{ANÁLISIS BIVARIADO POR PRUEBA DE HIPÓTESIS}

Se evidenció asociación estadísticamente significativa entre la NUSFPS con el sexo, la edad, el nivel educativo, la pareja estable, la región, tener algún problema de salud crónico, tener alguna discapacidad y tener seguro de salud (Tabla 2).

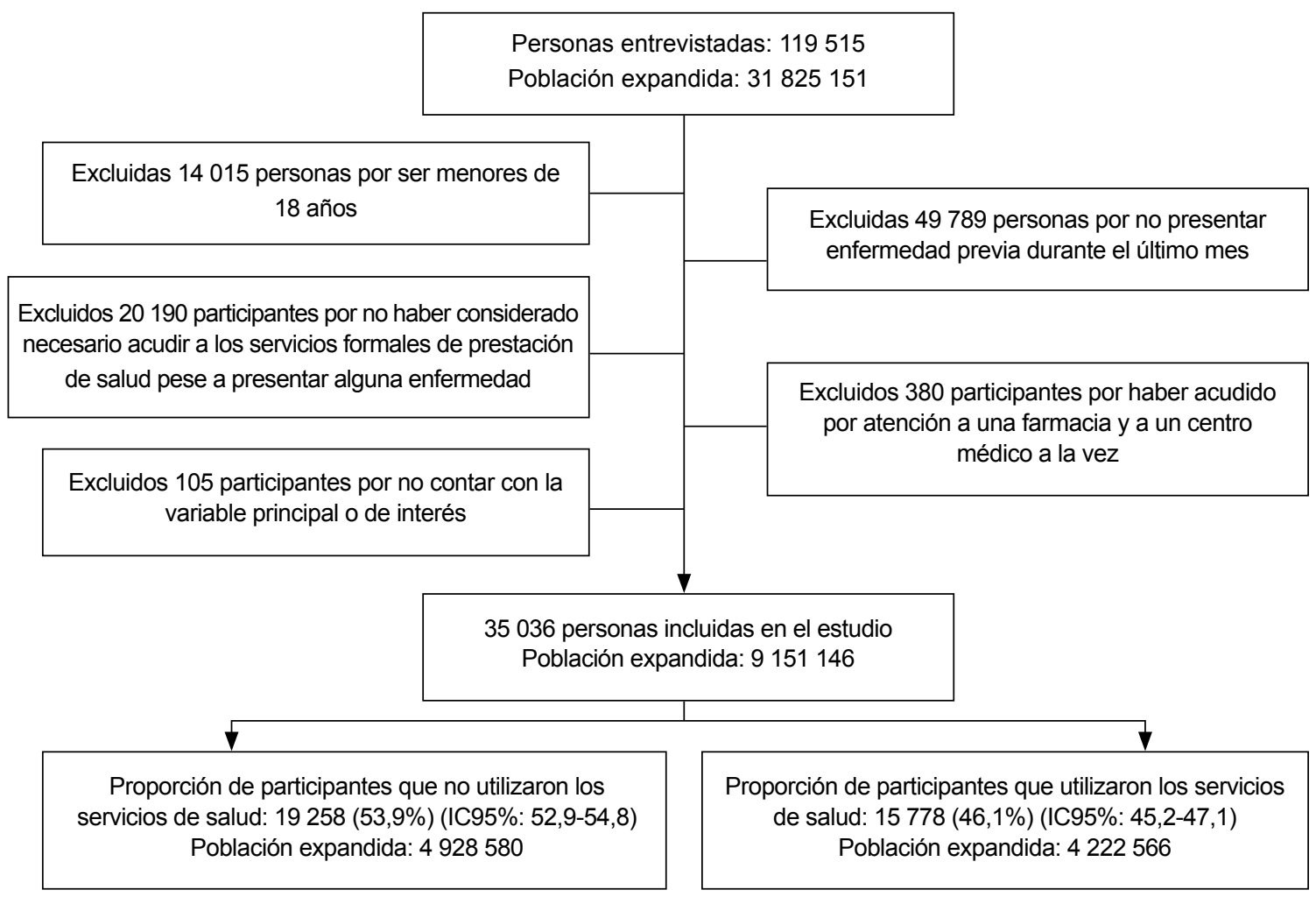

Figura 1. Flujograma de la selección de participantes incluidos en el análisis, Encuesta Nacional de Hogares (ENAHO) 2015. 
Tabla 1. Características generales de las personas adultas que presentaron algún síntoma o malestar, enfermedad, recaída de enfermedad crónica o accidente en Perú

\begin{tabular}{|c|c|c|c|}
\hline \multirow{2}{*}{ Características } & \multirow{2}{*}{$\begin{array}{c}\begin{array}{c}\text { Frecuencia } \\
\text { absoluta }\end{array} \\
\mathbf{n}\end{array}$} & \multicolumn{2}{|c|}{$\begin{array}{l}\text { Proporción } \\
\text { ponderada* }\end{array}$} \\
\hline & & $\%$ & (IC95\%) \\
\hline \multicolumn{4}{|l|}{ Sexo } \\
\hline Mujer & 20540 & 59,6 & $(59,0-60,1)$ \\
\hline Varón & 14496 & 40,4 & $(39,0-41,0)$ \\
\hline \multicolumn{4}{|l|}{ Edad } \\
\hline Promedio (IC95\%) & & \multicolumn{2}{|c|}{$48,1(47,8-48,4)$} \\
\hline 18 a 29 años & 6689 & 19,4 & $(18,8-20,0)$ \\
\hline 30 a 39 años & 5937 & 16,8 & $(16,2-17,4)$ \\
\hline 40 a 59 años & 12474 & 35,3 & $(34,6-36,0)$ \\
\hline 60 a más años & 9936 & 28,5 & $(27,7-29,3)$ \\
\hline \multicolumn{4}{|l|}{ Estado civil } \\
\hline Soltero & 5952 & 18,8 & $(18,2-19,4)$ \\
\hline Conviviente & 9425 & 25,4 & $(24,6-26,2)$ \\
\hline Casado & 13017 & 36,2 & $(35,4-37,1)$ \\
\hline Viudo & 3120 & 8,9 & $(8,5-9,3)$ \\
\hline Divorciado/separado & 3522 & 10,7 & $(10,2-11,1)$ \\
\hline \multicolumn{4}{|l|}{ Nivel educativo } \\
\hline Sin nivel & 3795 & 9,3 & $(8,9-9,8)$ \\
\hline Primaria $^{\dagger}$ & 12344 & 31,7 & $(31,0-32,5)$ \\
\hline Secundaria $^{\dagger}$ & 11385 & 35,5 & $(34,7-36,3)$ \\
\hline $\mathrm{SNU}^{\dagger}$ & 3392 & 10,8 & $(10,3-11,3)$ \\
\hline SUP $^{\dagger}$ & 4116 & 12,7 & $(12,0-13,4)$ \\
\hline \multicolumn{4}{|l|}{ Región } \\
\hline Lima Metropolitana & 3879 & 28,6 & $(27,0-30,1)$ \\
\hline Costa & 9008 & 21,5 & $(20,2-22,9)$ \\
\hline Sierra & 15215 & 38,4 & $(36,8-40,1)$ \\
\hline Selva & 6934 & 11,5 & $(10,6-12,4)$ \\
\hline \multicolumn{4}{|l|}{ Problema de salud } \\
\hline No & 13510 & 38,3 & $(37,4-39,1)$ \\
\hline Sí & 21526 & 61,7 & $(60,9-62,6)$ \\
\hline \multicolumn{4}{|l|}{ Discapacidad } \\
\hline No & 32310 & 92,5 & $(92,0-92,9)$ \\
\hline Sí & 2726 & 7,5 & $(7,1-8,0)$ \\
\hline \multicolumn{4}{|l|}{ Servicios de salud } \\
\hline No & 19258 & 53,9 & $(52,9-54,8)$ \\
\hline Sí & 15778 & 46,1 & $(45,2-47,1)$ \\
\hline \multicolumn{4}{|l|}{ Seguro de salud } \\
\hline Sin seguro & 8083 & 24,4 & $(23,7-25,2)$ \\
\hline SIS & 17874 & 45,3 & $(44,3-46,2)$ \\
\hline EsSalud & 7860 & 25,7 & $(24,8-26,6)$ \\
\hline FFAAPP & 412 & 1,6 & $(1,3-1,9)$ \\
\hline Seguro privado & 807 & 3,0 & $(2,7-3,4)$ \\
\hline
\end{tabular}

Datos provenientes de la Encuesta Nacional de Hogares (ENAHO) 2015 Tamaño muestral $=35036$

* Se incluyeron los pesos y el efecto de diseño propio del muestreo complejo de la encuesta.

† Hace referencia a instrucción superior universitaria, no universitaria, secundaria o primaria completas o incompletas.

SNU: Superior no universitario, SUP: Superior universitario o posgrado SIS: Seguro Integral de Salud, EsSalud: Seguro Social, FFAAPP: Fuerzas armadas y policiales
Tabla 2. Proporción de no utilización de los servicios formales de prestación de salud en personas adultas que presentaron algún síntoma o malestar, enfermedad, recaída de enfermedad crónica o accidente en Perú

\begin{tabular}{|c|c|c|c|c|}
\hline \multirow[t]{2}{*}{ Características } & \multirow{2}{*}{$\begin{array}{c}\text { Usuarios } \\
\text { n }\end{array}$} & \multicolumn{2}{|c|}{$\begin{array}{l}\text { Proporción } \\
\text { ponderada* }\end{array}$} & \multirow[t]{2}{*}{ Valor $p^{\S}$} \\
\hline & & $\% \pi$ & (IC95\%) & \\
\hline Sexo & & & & $<0,001$ \\
\hline Mujer & 10691 & 51,3 & $(50,2-52,4)$ & \\
\hline Varón & 8567 & 57,6 & $(56,4-58,8)$ & \\
\hline Edad & & & & $<0,001$ \\
\hline 18 a 29 años & 3954 & 58,8 & $(57,0-60,6)$ & \\
\hline 30 a 39 años & 3272 & 55,9 & $(54,0-57,7)$ & \\
\hline 40 a 59 años & 7024 & 55,1 & $(53,7-56,4)$ & \\
\hline 60 a más años & 5008 & 47,8 & $(46,3-49,3)$ & \\
\hline Nivel educativo & & & & $<0,001$ \\
\hline Sin Nivel & 2252 & 59,4 & $(57,1-61,6)$ & \\
\hline Primaria $\|$ & 6889 & 55,7 & $(54,3-57,0)$ & \\
\hline Secundaria $\|$ & 6308 & 54,1 & $(52,7-55,5)$ & \\
\hline SUP॥ & 3806 & 48,8 & $(47,1-50,5)$ & \\
\hline Pareja estable & & & & 0,169 \\
\hline $\mathrm{No}^{+}$ & 7148 & 54,5 & $(53,1-55,9)$ & \\
\hline Sí $\neq$ & 12110 & 53,4 & $(52,4-54,5)$ & \\
\hline Región & & & & $<0,001$ \\
\hline Lima Metropolitana & 1564 & 41,6 & $(39,5-43,7)$ & \\
\hline Costa & 4908 & 54,4 & $(52,6-56,2)$ & \\
\hline Sierra & 8983 & 61,9 & $(60,6-63,2)$ & \\
\hline Selva & 3803 & 56,5 & $(54,5-58,5)$ & \\
\hline Problema de salud & & & & $<0,001$ \\
\hline No & 8008 & 59,2 & $(57,9-60,5)$ & \\
\hline Sí & 11250 & 50,6 & $(49,4-51,7)$ & \\
\hline Discapacidad & & & & 0,102 \\
\hline No & 17792 & 54,0 & $(53,0-55,0)$ & \\
\hline Sí & 1466 & 51,8 & $(49,1-54,4)$ & \\
\hline Seguro de salud & & & & $<0,001$ \\
\hline Sin seguro & 5975 & 71,2 & $(69,6-72,8)$ & \\
\hline SIS & 9483 & 53,8 & $(52,6-55,0)$ & \\
\hline EsSalud & 3339 & 40,4 & $(38,8-42,0)$ & \\
\hline FFAAPP & 153 & 38,7 & $(32,2-45,6)$ & \\
\hline Seguro privado & 308 & 36,7 & $(32,2-41,4)$ & \\
\hline
\end{tabular}

Datos provenientes de la Encuesta Nacional de Hogares (ENAHO) 2015 Tamaño muestral $=35036$

* Se incluyeron los pesos y el efecto de diseño propio del muestreo complejo de la encuesta.

† Incluye las categorías: soltero, viudo, divorciado/separado.

* Incluye las categorías: conviviente y casado.

$\S$ Hace referencia a la significancia estadística obtenida a partir de la comparación de las proporciones entre las categorías de las variables considerando el muestreo complejo de la encuesta.

" Hace referencia a instrucción superior universitaria, no universitaria, secundaria o primaria completas o incompletas.

" Proporción ponderada de no utilización de los servicios de salud de acuerdo a cada categoría

SUP: Superior universitario o posgrado

SIS: Seguro Integral de Salud, EsSalud: Seguro Social, FFAAPP: Fuerzas armadas y policiales 


\section{MODELOS LINEALES GENERALIZADOS: CRUDOS Y AJUSTADOS}

En el análisis crudo se evidenció mayor prevalencia de NUSFPS en el sexo masculino, en las personas menores de 60 años, en aquellos con un nivel educativo diferente del superior, el residir en una región distinta a Lima Metropolitana, y no contar con seguro de salud o estar asegurado al SIS en comparación a aquellos usuarios de EsSalud. El tener algún problema de salud crónico, se asoció a una menor prevalencia de NUSFPS.
En el análisis ajustado se mantuvieron asociadas a una mayor prevalencia de NUSFPS: el sexo masculino; el tener de 18 a 29 años, 30 a 39 años o 40 a 59 años (en comparación a tener de 60 a más años); el no tener grado de instrucción, contar con educación primaria o secundaria (en comparación con nivel educativo superior); el residir en resto de la costa, sierra o selva (en comparación con residir en Lima Metropolitana); y el no estar asegurado o estar afiliado al SIS (con respecto a estar afiliado a EsSalud). Por otro lado, el tener algún problema de salud crónico, se asoció a una menor prevalencia de NUSFPS (Tabla 3).

Tabla 3. Modelos lineales generalizados de familia Poisson crudo y ajustado para muestras complejas para el desenlace de no utilización de los servicios formales de prestación de salud en personas adultas en Perú

\begin{tabular}{|c|c|c|c|c|c|c|}
\hline \multirow{2}{*}{ Características } & \multicolumn{3}{|c|}{ Modelo crudo* } & \multicolumn{3}{|c|}{ Modelo Ajustado* } \\
\hline & RP & (IC95\%) & Valor $p$ & RPa & (IC95\%) & Valor $p$ \\
\hline \multicolumn{7}{|l|}{ Sexo } \\
\hline Mujer & Ref. & & & Ref. & & \\
\hline Varón & 1,12 & $(1,10-1,15)$ & $<0,001$ & 1,12 & $(1,09-1,15)$ & $<0,001$ \\
\hline \multicolumn{7}{|l|}{ Edad } \\
\hline De 60 a más años & Ref. & & & Ref. & & \\
\hline De 18 a 29 años & 1,23 & $(1,18-1,28)$ & $<0,001$ & 1,17 & $(1,12-1,23)$ & $<0,001$ \\
\hline 30 a 39 años & 1,17 & $(1.12-1.22)$ & $<0,001$ & 1,17 & $(1,12-1,23)$ & $<0,001$ \\
\hline 40 a 59 años & 1,15 & $(1,11-1,19)$ & $<0,001$ & 1,15 & $(1,11-1,19)$ & $<0,001$ \\
\hline \multicolumn{7}{|l|}{ Nivel educativo } \\
\hline Superior $\S$ o posgrado & Ref. & & & Ref. & & \\
\hline Sin nivel & 1,22 & $(1,16-1,28)$ & $<0,001$ & 1,23 & $(1,16-1,30)$ & $<0,001$ \\
\hline Primaria $\S$ & 1,14 & $(1,10-1,19)$ & $<0,001$ & 1,10 & $(1,06-1,15)$ & $<0,001$ \\
\hline Secundaria $\S$ & 1,11 & $(1,07-1,15)$ & $<0,001$ & 1,05 & $(1,01-1,10)$ & 0,008 \\
\hline \multicolumn{7}{|l|}{ Pareja estable } \\
\hline $\mathrm{No}^{+}$ & Ref. & & & Ref. & & \\
\hline Sí ‡ & 0,98 & $(0,95-1,01)$ & 0,167 & 0,98 & $(0,95-1,08)$ & 0,145 \\
\hline \multicolumn{7}{|l|}{ Región } \\
\hline Lima Metropolitana & Ref. & & & Ref. & & \\
\hline Costa & 1,31 & $(1,23-1,39)$ & $<0,001$ & 1,24 & $(1,17-1,31)$ & $<0,001$ \\
\hline Sierra & 1,49 & $(1,41-1,57)$ & $<0,001$ & 1,38 & $(1,31-1,46)$ & $<0,001$ \\
\hline Selva & 1,36 & $(1,28-1,44)$ & $<0,001$ & 1,25 & $(1,18-1,33)$ & $<0,001$ \\
\hline \multicolumn{7}{|l|}{ Problema de salud crónico } \\
\hline No & Ref. & & & Ref. & & \\
\hline Sí & 0,85 & $(0,83-0,88)$ & $<0,001$ & 0,92 & $(0,89-0,95)$ & $<0,001$ \\
\hline \multicolumn{7}{|l|}{ Discapacidad } \\
\hline No & Ref. & & & Ref. & & \\
\hline Sí & 0,96 & $(0,91-1,01)$ & 0,109 & 0,99 & $(0,94-1.04)$ & 0,627 \\
\hline \multicolumn{7}{|l|}{ Seguro de salud } \\
\hline EsSalud & Ref. & & & Ref. & & \\
\hline Sin seguro & 1,76 & $(1,69-1,84)$ & $<0,001$ & 1,59 & $(1,52-1,66)$ & $<0,001$ \\
\hline SIS & 1,33 & $(1,28-1,39)$ & $<0,001$ & 1,16 & $(1,11-1,22)$ & $<0,001$ \\
\hline FFAAPP & 0,96 & $(0,80-1,14)$ & 0,635 & 1,02 & $(0,87-1,23)$ & 0,787 \\
\hline Seguro privado & 0,91 & $(0,80-1,04)$ & 0,155 & 0,95 & $(0,83-1,08)$ & 0,425 \\
\hline
\end{tabular}

Datos provenientes de la Encuesta Nacional de Hogares (ENAHO) 2015

Tamaño muestral = 35036

FFAAPP: Fuerzas armadas y policiales

Se realizó un modelo lineal generalizado de familia Poisson con link log considerando el efecto de diseño y las ponderaciones del muestreo complejo de la encuesta.

EsSalud: Seguridad Social, SIS: Seguro integral de salud, FFAAPP: Fuerzas armadas y policiales

† Incluye las categorías: soltero, viudo, divorciado/separado.

‡ Incluye las categorías: conviviente y casado.

$\S$ Hace referencia a instrucción superior universitaria, no universitaria, secundaria o primaria completas o incompletas 


\section{DISCUSIÓN}

Los hallazgos del presente estudio muestran que, en Perú, más de la mitad de las personas adultas no utilizaron los servicios formales de salud a pesar de presentar algún síntoma, malestar, enfermedad, recaída de enfermedad crónica o accidente, y de considerar que era necesario recibir atención médica. En Latinoamérica, dos estudios que incluyeron población de Colombia, Brasil y México reportaron prevalencias de NUSFPS de $25,7 \%, 57,2 \%$ y 88,6\%: respectivamente ${ }^{(5,6)}$. En Europa, la prevalencia de NUSFPS bucal en España, Suecia y Dinamarca osciló entre 65,7 y $20 \%$, respectivamente ${ }^{(7,8)}$. Nosotros creemos que este bajo porcentaje se atribuiría a que estos países cuentan con un mejor desarrollo y cultura en salud. A nivel nacional, un estudio realizado en el año 2014, halló que solo el $32,8 \%$ de los pacientes con trastornos de salud mental pudo utilizar un servicio de salud especializado ${ }^{(9)}$. Asimismo, el INEI reportó la prevalencia de utilización de los servicios de salud por haber padecido de algún problema de salud crónico y no crónico desde el año 2004 , las cuales fueron de 38,0 y $38,7 \%$, respectivamente; este porcentaje se ha incrementado de forma progresiva hasta que en el año 2015 alcanzó porcentajes de 41,3 y $53,5 \%$, correspondientemente ${ }^{(10)}$.

Se halló una mayor prevalencia de NUSFPS en aquellos participantes de sexo masculino, lo cual concuerda con lo hallado en estudios realizados en Colombia, Brasil y Dinamarca ${ }^{(5,11,12)}$. Creemos que esta situación podría deberse al carácter predominantemente machista presente en la población peruana ${ }^{(13)}$; el varón tiende a trabajar durante largas jornadas, con reducido tiempo para el cuidado de su salud. También, se determinó que las personas jóvenes y adultas cuentan con mayor prevalencia de NUSFPS en comparación a los adultos mayores, lo cual concuerda con estudios previos ${ }^{(5,11)}$. Esta asociación se podría deber a múltiples factores, siendo el principal, la presencia de comorbilidades. En Colombia, aproximadamente el $24,8 \%$ de los adultos mayores padecían de múltiples enfermedades crónicas simultáneamente ${ }^{(14)}$.

Se halló que las personas con menor nivel educativo presentaron mayor prevalencia de NUSFPS. Ello es consistente con un estudio a nivel nacional se concluye que las personas con mayor nivel educativo acuden con mayor frecuencia a servicios de salud ambulatorios ${ }^{\left({ }^{15}\right)}$, y con una revisión sistemática halló una asociación positiva entre los años de educación y la utilización de servicios de salud (16).

Se halló que aquellas personas sin seguro de salud o afiliados al SIS tenían mayor prevalencia de NUSFPS comparado con las personas afiliadas a EsSalud. En México, el Seguro Popular, incrementa el número de consultas externas, lo cual contradice a lo hallado en Perú (17). El presente estudio cobra relevancia en los participantes que residen en zonas de sierra y selva, dado que otros estudios lo sitúan como un factor de riesgo para la NUSFPS ${ }^{(18)}$. La falta de médicos generales, especialistas, personal sanitario capacitado y atención rápida aumentaría la probabilidad de NUSFPS en esta población ${ }^{(19)}$.

Este estudio posee limitaciones. En primer lugar, el diseño transversal del estudio no permite evaluar causalidad; no obstante, el objetivo de la investigación se centró en la estimación de la frecuencia y factores asociados, situación que puede ser respondida por diseños transversales. En segundo lugar, al ser un análisis secundario de datos, la pregunta de investigación no ha sido formulada previo a la elaboración de los cuestionarios y a la ejecución del estudio primario, por lo que se han incluido en el análisis solo las variables que se encuentran en los cuestionarios de la ENAHO. En tercer lugar, la exclusión de las personas menores de 18 años podría producir un sesgo de selección, no obstante, no se evidenció diferencias en términos de sexo y región, entre las observaciones incluidas y las excluidas.

En conclusión, en Perú, una gran cantidad de personas que potencialmente requieren atención médica, no logran utilizar un servicio de salud, lo cual se asoció con condiciones demográficas y de aseguramiento.

Contribuciones de autoría: VABZ, MLU, EMB y EMH participaron de la concepción del estudio. VABZ, MLU, DUP, $E M B, E M M$ y EMH participaron en el diseño del estudio e interpretación de resultados. VABZ, DUP y EMM redactaron el borrador del manuscrito. VABZ, DUP y EMH realizaron el análisis de datos. Todos los autores hicieron la revisión crítica del manuscrito, aprobaron la versión a publicar y se responsabilizan del contenido del artículo.

Fuentes de financiamiento: el presente trabajo ha sido autofinanciado por los autores.

Conflictos de interés: los autores declaran no tener conflictos de interés con respecto a la publicación del presente artículo.

\section{REFERENCIAS BIBLIOGRÁFICAS}

1. O’Meara G, Ruiz F, Amaya JL. Impacto del aseguramiento sobre uso y gasto en salud en Colombia. Bogotá: CEJA; 2003.

2. Montastruc JL, Bondon-Guitton E, Abadie D, Lacroix I, Berreni A, Pugnet $\mathrm{G}$, et al. Pharmacovigilance, risks and ad- verse effects of self-medication. Thérapie. 2016;71(2):257-62. doi: 10.1016/j.therap.2016.02.012.

3. Alcalde-Rabanal JE, Lazo-González O, Nigenda G. Sistema de salud de Perú. Salud Publica Mex. 2011;53 Suppl 2:s243-54.
4. Acharya A, Vellakkal S, Taylor F, Masset E, Satija A, Burke M, et al. Impact of national health insurance for the poor and the informal sector in low-and middle-income countries. Systematic Review, London: The EPPI-Centre; 2012. 
5. Garcia-Subirats I, Lorenzo IV, Mogollón-Pérez AS, De Paepe P, da Silva MRF, Unger JP, et al. Determinantes del uso de distintos niveles asistenciales en el Sistema General de Seguridad Social en Salud y Sistema Único de Salud en Colombia y Brasil. Gac Sanit. 2014;28(6):480-8. doi: 10.1016/j.gaceta.2014.05.010.

6. Borges-Yáñez SA, Gómez-Dantés H. Uso de los servicios de salud por la población de 60 años y más en México. Salud Publica Mex. 1998;40(1):13-23.

7. Pizarro V, Ferrer M, Domingo-Salvany A, Benach J, Borrell C, Pont A, et al. The utilization of dental care services according to health insurance coverage in Catalonia (Spain). Community Dent Oral Epidemiol. 2009;37(1):78-84. doi: 10.1111/j.1600-0528.2008.00439.x.

8. Kronström M, Palmqvist S, Söderfeldt B, Vigild M. Utilization of dental health services among middle-aged people in Sweden and Denmark. Acta Odontol Scand. 2002;60(5):276-80.

9. Piazza M, Fiestas F. Prevalencia anual de trastornos y uso de servicios de salud mental en el Perú: resultados del estudio mundial de salud mental, 2005. Rev Peru Med Exp Salud Publica. 2014;31(1):30-8.
10. Instituto Nacional de Estadistica e Informática. Condiciones de vida en el Perú: Octubre-Noviembre-Diciembre 2015. [Internet]. Lima: INEI; 2015 [citado 15 de abril de 2017]. Disponible en: https://www.inei.gob.pe/ media/MenuRecursivo/boletines/ informe-tecnico-n01_condiciones-vida_octnovdic14.pdf

11. Mendoza-Sassi R, Béria JU, Barros AJ. Outpatient health service utilization and associated factors: a population-based study. Rev Saude Publica. 2003;37(3):372-8.

12. Krasnik A, Hansen E, Keiding N, Sawitz A. Determinants of general practice utilization in Denmark. Dan Med Bull. 1997;44(5):542-6.

13. Flake DF. Individual, family, and community risk markers for domestic violence in Peru. Violence Against Women. 2005;11(3):353-73.

14. Melguizo-Herrera E, Castillo-Ávila IY. Factores asociados al uso de servicios de atención primaria por adultos mayores de Cartagena, Colombia. Rev Salud Publica (Bogota). 2012;14(5):765-75.

15. Ypanaqué-Luyo P, Martins M. Uso de los servicios de salud ambulatorios en la población peruana. Rev Peru Med Exp Salud Publica. 2015;32(3):464-70.
16. Dias da Costa JS, Facchini LA. Utilização de serviços ambulatoriais em Pelotas: onde a população consulta e com que freqüência. Rev Saude Publica. 1997;31(4):360-9.

17. Nigenda G. El seguro popular de salud en México. Desarrollo y retos para el futuro. Washington (DC): Banco Interamericano de Desarrollo; 2005.

18. Liu X, Gao W, Yan H. Measuring and decomposing the inequality of maternal health services utilization in Western Rural China. BMC Health Serv Res. 2014;14:102. doi: 10.1186/1472-696314-102.

19. Ministerio de Salud. Segunda medición de las metas regionales de recursos humanos para la Salud, Perú 2007-2015 [Internet]. Lima: Dirección General de Gestión del Desarrollo de Recursos Humanos, Minsa; 2013. [citado 21 de abril de 2017]. Disponible en: http://www. minsa.gob.pe/dggdrh/libros/pdf/s1/I15.\%20Segunda\%20Medicion.pdf

Correspondencia: Edward Mezones-Holguin Dirección: Prolongación Primavera 2390, Monterrico, Santiago de Surco. Lima, Perú.

Teléfono: (+511) 3118888

Correoelectrónico:emezones@gmail.com

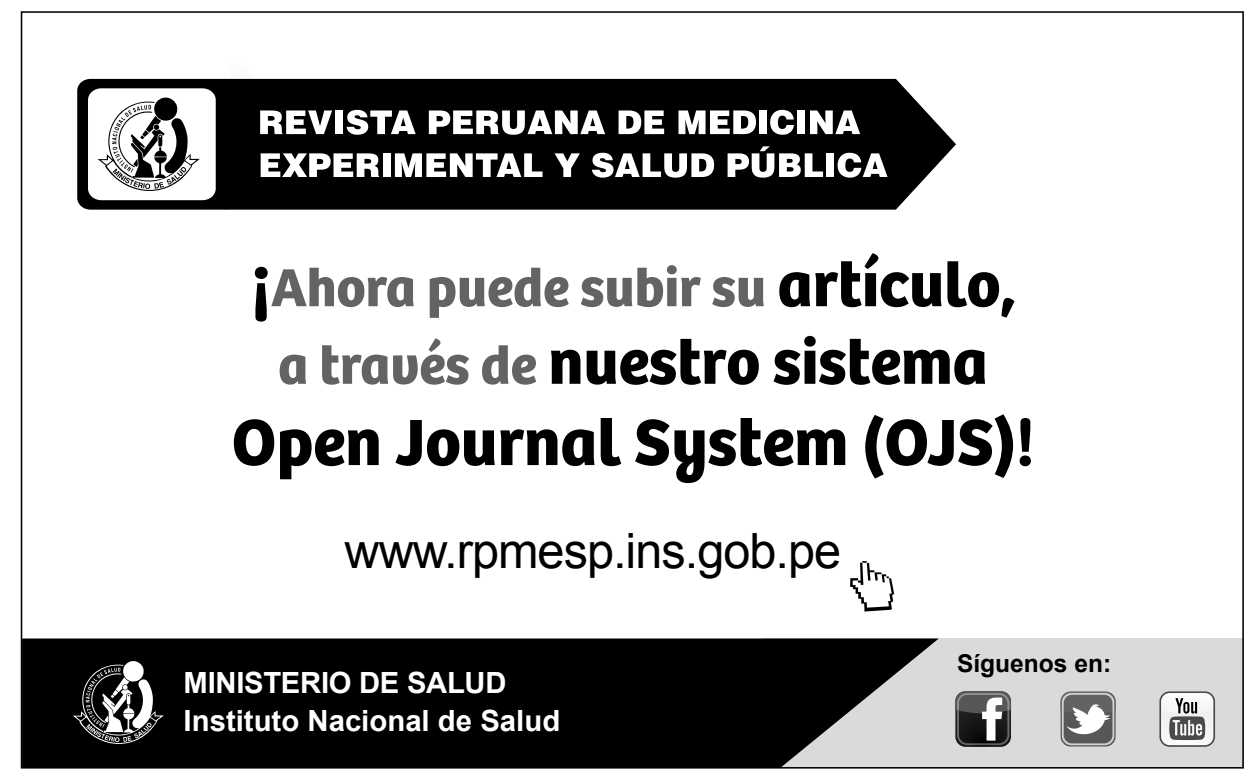

